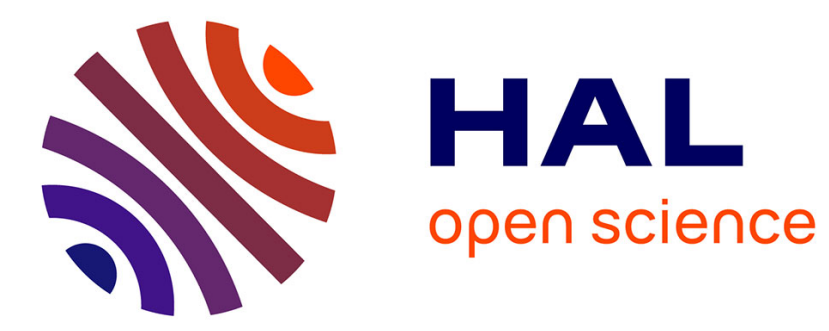

\title{
Photonic band-gap guidance in high-porosity luminescent porous silicon
}

Patrick Ferrand, D. Loi, R. Romestain

\section{To cite this version:}

Patrick Ferrand, D. Loi, R. Romestain. Photonic band-gap guidance in high-porosity luminescent porous silicon. Applied Physics Letters, 2001, 79 (19), pp.3017-3019. 10.1063/1.1414302 . hal00272392

\section{HAL Id: hal-00272392 \\ https://hal.science/hal-00272392}

Submitted on 15 Apr 2015

HAL is a multi-disciplinary open access archive for the deposit and dissemination of scientific research documents, whether they are published or not. The documents may come from teaching and research institutions in France or abroad, or from public or private research centers.
L'archive ouverte pluridisciplinaire HAL, est destinée au dépôt et à la diffusion de documents scientifiques de niveau recherche, publiés ou non, émanant des établissements d'enseignement et de recherche français ou étrangers, des laboratoires publics ou privés. 


\title{
Photonic band-gap guidance in high-porosity luminescent porous silicon
}

\author{
P. Ferrand, ${ }^{\text {a) }}$ D. Loi, and R. Romestain \\ Laboratoire de Spectrométrie Physique, Université J. Fourier Grenoble 1-CNRS UMR 5588, BP 87, \\ F-38402 Saint Martin d'Hères, France
}

(Received 23 July 2001; accepted for publication 29 August 2001)

\begin{abstract}
We demonstrate a nanoporous silicon (nPS) multilayered structure, offering an efficient photonic band-gap (PBG) single-mode guidance in a high-porosity (i.e., low index) layer in the near-infrared. We show the good agreement between the calculated band structure and the measured spectral range of efficient guiding. We discuss the different kinds of guided modes that exist within the structure, and verify that only one PBG mode can be guided efficiently. Quantitative measurements of the losses are performed using the room-temperature photoluminescence of nPS. (C) 2001 American Institute of Physics. [DOI: 10.1063/1.1414302]
\end{abstract}

The strong research effort devoted to photonic band-gap (PBG) materials ${ }^{1}$ is motivated by their unique property to allow an efficient guiding of light even across tight bends, ${ }^{2}$ opening the way to the realization of compact integrated photonic circuits. This justifies the urgent requirement for low-cost materials, whose optical index can be modulated at a submicronic scale. Moreover, light emitting materials are preferred, due to the convenience they bring while performing optical characterizations. ${ }^{3}$

In this context, nanoporous silicon (nPS) is a serious candidate, thanks to its optical index that can be tailored by changing the porosity. Efficient optical microstructures have been already demonstrated, using submicronic index modulations in depth ${ }^{4}$ or in the plane. ${ }^{5}$ In addition, high-porosity nPS layers present the unique property of photoluminescence (PL) at room temperature. ${ }^{6}$ Unfortunately, due to their low index, they cannot channel the emitted light by step-index guidance. ${ }^{7}$ In this letter, we demonstrate an nPS multilayered structure, offering an efficient single-mode PBG guidance in a high porosity luminescent layer.

Our sample was realized by etching successive layers of different porosities. ${ }^{4}$ We used low-doped $p$ type (100) silicon substrate of resistivity $4-7 \Omega \mathrm{cm}$, with a thickness of only $100 \mu \mathrm{m}$ to allow perfect cleaved edges. A HF: $\mathrm{H}_{2} \mathrm{O}: \mathrm{C}_{2} \mathrm{H}_{5} \mathrm{OH}$ (35:35:30) solution at room temperature, and current densities of 16.6 and $333 \mathrm{~mA} \mathrm{~cm}^{-2}$ were used, giving rise to respective porosities of $58 \%$ and $72 \%$. After rinsing, an anodic oxidation was performed, in a $1 \mathrm{M} \mathrm{HCl}$ water solution, using a current density of $1 \mathrm{~mA} \mathrm{~cm}^{-2}$, in order to increase and stabilize the PL. ${ }^{8}$

Two kinds of optical characterizations have been performed on the same optical bench described in Fig. 1(a). Transmittance spectra have been performed by end-fire coupling, using the white light of a tungsten lamp, and dispersion-free microscope objectives. ${ }^{9}$ PL measurements were performed by pumping using an $\mathrm{Ar}^{+}$ion laser $(\lambda$ $=0.4579 \mu \mathrm{m})$ by imaging a slit using a microscope objective, giving rise to a rectangular spot of size $130 \mu \mathrm{m}$ $\times 400 \mu \mathrm{m}$ of power density $0.6 \mathrm{~W} \mathrm{~cm}^{-2}$, parallel and at a distance $L$ from the output edge. The focusing of the objec-

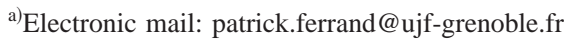

tive denoted C in Fig. 1(a) was finely tuned in order to select the light outcoming from the plane of the output edge.

The detection setup, based on the simultaneous imaging on a camera and on the entrance slit of a monochromator, has been described in a previous paper. ${ }^{9}$ In addition, normal incidence absolute reflectance spectra have been performed using a two-beam Perkin-Elmer Lambda 9 spectrophotometer.

Conventional step-index guidance needs a slab of index $n_{g}$ surrounded by a medium (cladding) having a lower index $n_{\text {clad }}$. The confinement of light occurs for propagation constants $k_{x}$ verifying $k n_{\text {clad }}<k_{x}<k n_{g}$, where $k$ is modulus of the vacuum wave vector, thanks to the total internal reflection (TIR) and thus to the exponential decay of field outside the slab. The PBG guidance does not need any TIR, but a Bragg reflection on a periodic surrounding medium. ${ }^{10}$ At a given optical frequency $\omega$, the only requirement is that this medium possesses a range of forbidden values for $k_{x}$. In this case, light cannot propagate in the periodic medium and vanishes after a number of periods that depends on the index contrast.

As illustrated in Fig. 1(b), our structure is constituted by a periodic stacking of 4.5 pairs of alternating layers of high $(\mathrm{H})$ and low $(\mathrm{L})$ indices $\left(n_{\mathrm{H}}=1.55, n_{\mathrm{L}}=1.2\right.$, thicknesses $t_{\mathrm{H}}$ $=0.186 \mu \mathrm{m}, t_{\mathrm{L}}=0.576 \mu \mathrm{m}$ ), and an additional layer of index $n_{g}=n_{\mathrm{L}}$, of thickness $t_{g}=2 t_{\mathrm{L}}$, localized at the top. At a given frequency, guiding can occur in this last layer if the two following conditions are verified simultaneoulsy: (i) TIR at the top air interface, i.e., $k<k_{x}<k n_{g}$; (ii) Bragg reflection
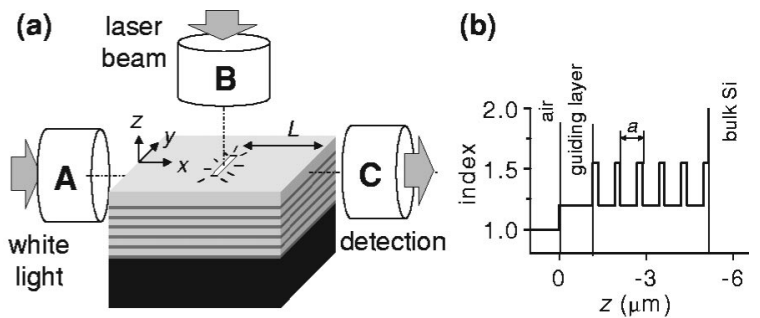

FIG. 1. (a) Scheme of the multilayered nPS microstructure, and experimental setup used for optical characterizations. Microscope objectives are designated by capital letters. A and $\mathrm{C}$ are used for white-light transmission measurement; PL measurements need B and C; (b) index profile of the sample. 


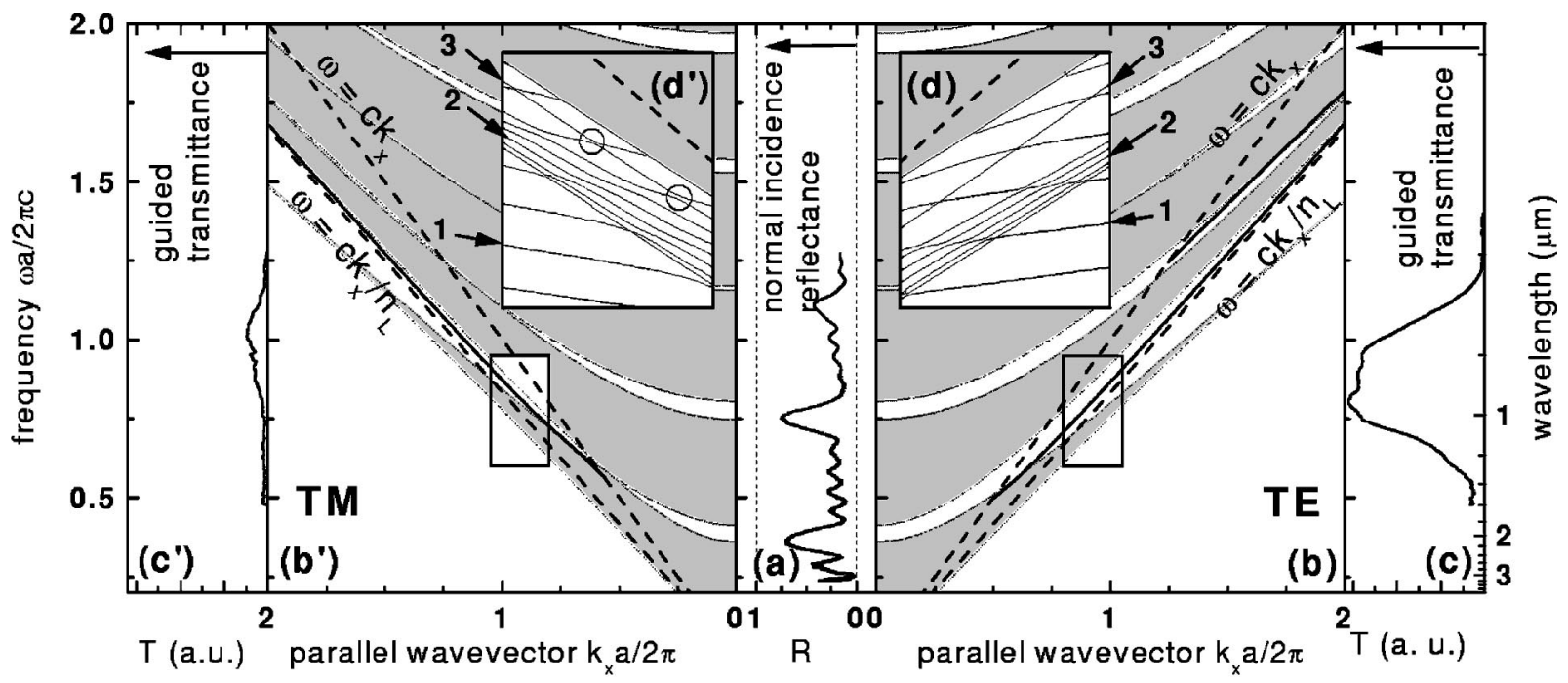

FIG. 2. (a) Normal-incidence absolute reflectance experimental spectrum; $\left(\mathrm{b}, \mathrm{b}^{\prime}\right)$ projected band structure for the infinite multilayer. White regions correspond to evanescent states. Dashed lines $\omega=c k_{x}$ and $\omega=c k_{x} / n_{\mathrm{L}}$ indicate the boundary values of $k_{x}$ in order to have a TIR at the top air interface. The black lines inside some gaps correspond to PBG guided modes. $\left(\mathrm{c}, \mathrm{c}^{\prime}\right)$ Guided transmittance experimental spectra, plotted for both polarizations using the same scale; $\left(\mathrm{d}, \mathrm{d}^{\prime}\right)$ modes calculated for the real structure in the range represented by the rectangle, showing all the types of guided modes present in the structure: (1) substrate guided modes, (2) high index multilayers guided modes, (3) PBG guided mode. The circles show the splitting, as a proof of the coupling between PBG and substrate guided modes.

on the bottom multilayer, i.e., $k_{x}$ is forbidden. We define $a=t_{\mathrm{H}}+t_{\mathrm{L}}$. The index of the substrate is $n_{\mathrm{sub}}=3.5$.

Limiting ourselves first to the simple case of an infinite multilayer, we have calculated and plotted its projected band structure as a function of $k_{x}$, for all possible values of $k_{z}$, as shown in Figs. 2(b) and 2(b'), respectively for TE and TM polarizations. ${ }^{11}$ Propagating states exist in shaded regions while white regions allow only evanescent states. In addition, we have reported the lines $\omega=c k_{x}$ and $\omega=c k_{x} / n_{\mathrm{L}}$, between which the condition of TIR at the top air interface is verified. The normal-incidence absolute reflectance experimental spectrum, plotted in Fig. 2(a), shows the agreement between calculated gaps and high reflectivity ranges. Of course in our structure, the reflectance (less than 80\%) is limited by the low number of periods. However, at large incidence, i.e., for large $k_{x}$, the reflectance becomes higher, ${ }^{12}$ due to high reflection coefficients at each interface (excepted near the Brewster incidence for TM polarization), so that even a finite structure with a low number of periods possesses forbidden frequencies very close to those calculated in infinite structures.

Then we calculated the band structure of the real structure, constituted by the guiding layer, the finite multilayer, and a substrate of an arbitrary thickness. We considered only the bands below the air line, and in order to keep a clear figure, we have only reported the major changes compared to the infinite multilayer case, i.e., the apparition of modes, plotted using a black line in Figs. 2(b) and 2( $\left.\mathrm{b}^{\prime}\right)$, inside the first TE and TM gaps, and in the second TE gap. Calculations of field distribution for these modes, not plotted here, show that the field is confined in the guiding layer.

Guided transmittance spectra, performed on a sample of length $1.88 \mathrm{~mm}$ and plotted in Figs. 2(c) and 2( $\left.\mathrm{c}^{\prime}\right)$, show that theses modes correspond to an efficient guiding. One should expect a relation between the cutoff frequencies and the disappearance of the mode. This appears clearly for the lowfrequency cutoff for $\omega a / 2 \pi c \sim 0.5$. The high-frequency cut- off appears earlier, due to the intrinsic absorption losses in nPS in the visible. ${ }^{13}$ In addition, strong polarization effects appear: the guiding efficiency is typically six times smaller in TM than in TE polarization.

In order to illustrate the main sources of guiding losses, Figs. 2(d) and 2(d') plotted all the modes given by the calculation of the real structure, in the range given by the rectangle in Figs. 2(b) and 2(b'). Three types of modes have been indexed and indicated by arrows. Type 1 modes, guided by TIR, are localized mainly within the substrate and exist between the air line and substrate line $\left(\omega=c k_{x} / n_{\text {sub }}\right.$, not plotted here). In our calculations, the substrate thickness was arbitrary fixed to $2 a$, in order to give well-spaced modes on the figure. In the real case, the substrate can be treated as

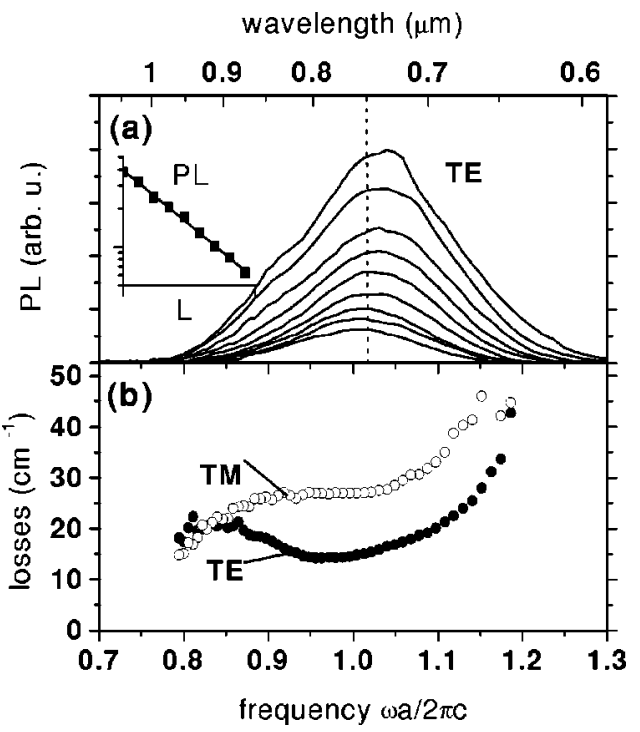

FIG. 3. (a) Set of PL spectra obtained by varying the distance $L$ between the exciting spot and the output edge. For each frequency, the decay of PL is fitted by an exponential law, as shown by the inset in log-scale; (b) deduced losses vs frequency for TE and TM polarizations. 
infinitely thick, giving rise to a continuum of states. The five type 2 modes are guided by TIR in the five layers of index $n_{1}$ surrounded by layers of index $n_{2}$ and constituting a multichannel waveguide, giving rise to a set of coupled modes. ${ }^{12}$ In the infinite multilayer they show up as a continuum. The type 3 is our PBG-guided mode.

Except absorption and scattering, the only source of losses for light guided in this mode is the leakage into the substrate, due to the finite multilayer. In other words, type 1 and type 3 modes could be coupled together, giving rise to an anticrossing in the band structure. Such anticrossing is not visible in Fig. 2(d), but appears clearly in Fig. 2(d') (circles), especially at low frequency. This is a qualitative justification for the higher losses in TM, that can be attributed to the smaller performance of Bragg reflectors in this polarization. ${ }^{12}$ In addition, it is reasonable to think that the transmitted light has not been guided by type 2 modes, because they appear very lossy, compared to type 3 , due to the vicinity of the substrate. Moreover, we have calculated that they would give a cutoff at a very low frequency. In these conditions, only one mode can be efficiently guided for reduced frequencies between 0.5 and 1.25.

Finally, we have measured the PL emitted by the edge of our structure. The use of a movable light emitter within the structure avoids the difficulties arising when incoupling through the sample edge. Due to the fact that only high porosity (i.e., low index) nPS layers are luminescent, ${ }^{6}$ and that the pump is absorbed in depth, the PL is assumed to be emitted only by the guiding layer. We measured a set of PL spectra for both polarizations, for several values of the propagation length $L$ between 0 and $1.2 \mathrm{~mm}$, as shown in TE in Fig. 3(a), taking care to remove the contribution of the divergence of the guided beam to the decay. ${ }^{14}$ The inset shows the values of PL (black squares) taken along the dotted line plotted in log-scale vs $L$, and the fit by an exponential decay (solid line), allow to obtain the losses with a high accuracy. The deduced losses for both polarizations are plotted in Fig. 3(b).

The obtained values $\left(15 \mathrm{~cm}^{-1}\right.$ at $\left.\lambda=0.780 \mu \mathrm{m}, \mathrm{TE}\right)$ are compatible with the known values of absorption in high porosity $\mathrm{nPS},{ }^{13}$ if we take into account the reduction of losses due to the partial oxidation of the structure step. ${ }^{8}$ The polar- ization effects mentioned above are confirmed, with typical additional losses of $12 \mathrm{~cm}^{-1}$ for TM polarization. The shape of the TE curve illustrates the competition between absorption losses that dominate for $\omega a / 2 \pi c>1.1$, and guiding losses for $\omega a / 2 \pi c<0.9$. Finally, we would stress the fact that the porous nature of nPS allows the use of other impregnated emitters. ${ }^{15}$

In summary, we demonstrated an nPS multilayered structure, offering an efficient single-mode PBG guidance in a high-porosity layer. We also performed quantitative measurements of the losses using the PL of nPS.

The authors would like to thank A. Bsiesy, S. Setzu, and J. C. Vial for their fruitful advice. D.L. is also with University of Cagliari, Italy. This work was partially supported by the Centre National de la Recherche Scientifique (CNRS) in the framework of the Ultimatech program.

${ }^{1}$ See, e.g., special issue of J. Lightwave Technol. 17, 1928 (1999).

${ }^{2}$ E. Chow, S. Y. Lin, J. R. Wendt, S. G. Johnson, and J. D. Joannopoulos, Opt. Lett. 26, 286 (2001).

${ }^{3}$ D. Labilloy, H. Benisty, C. Weisbuch, T. Krauss, R. M. De La Rue, V. Bardinal, R. Houdré, U. Oesterle, D. Cassagne, and C. Jouanin, Phys. Rev. Lett. 79, 4147 (1997).

${ }^{4}$ S. Setzu, P. Ferrand, and R. Romestain, Mater. Sci. Eng., B 69-70, 34 (2000).

${ }^{5}$ P. Ferrand, R. Romestain, and J. C. Vial, Phys. Rev. B 63, 115106 (2001).

${ }^{6}$ L. T. Canham, Appl. Phys. Lett. 57, 1046 (1990).

${ }^{7}$ Even if Araki et al. demonstrated an edge-emitting device, the guiding of the photoluminescence (PL) was not obvious; see M. Araki, H. Koyama, and N. Koshida, Appl. Phys. Lett. 68, 2999 (1996).

${ }^{8}$ A. Bsiesy, J. C. Vial, F. Gaspard, R. Hérino, M. Ligeon, F. Müller, R. Romestain, A. Wasiela, A. Halimaoui, and G. Bomchil, Surf. Sci. 254, 195 (1991).

${ }^{9}$ P. Ferrand and R. Romestain, Appl. Phys. Lett. 77, 3535 (2000).

${ }^{10}$ P. Yeh and A. Yariv, Opt. Commun. 19, 427 (1976).

${ }^{11}$ S. G. Johnson and J. D. Joannopoulos, The MIT Photonic-Bands Package, http://ab-initio.mit.edu/mpb/.

${ }^{12} \mathrm{P}$. Yeh, Optical Waves in Layered Media (Wiley, New York, 1988).

${ }^{13}$ G. Lérondel, F. Madéore, R. Romestain, and F. Müller, Thin Solid Films 366, 216 (2000).

${ }^{14}$ In our case, the width of the spot is $400 \mu \mathrm{m}$ and is also the effective width of output edge that can be analyzed. In our setup, it can be shown that the divergence of the beam gives rise to a decay of the measured intensity vs $L$, linear for $L<1.7 \mathrm{~mm}$. For this last value, the attenuation is $50 \%$.

${ }^{15}$ S. Setzu, P. Solsona, S. Létant, R. Romestain, and J. C. Vial, Eur. Phys. J.: Appl. Phys. 7, 59 (1999); H. A. Lopez and P. M. Fauchet, Appl. Phys. Lett. 77, 3704 (2000). 